strategic pillar on diversity and inclusion in undergraduate research was the basis for the model. The hope in presenting this pilot is to provide the undergraduate research community with an innovative model to increase participation in undergraduate research by offering diverse and inclusive research experiences.

The Multicultural Center UAs engage in hands-on diversity and inclusion work, which provides them with a platform for later development of their own research projects. A central understanding of these positions is that the students' research projects may represent diversity in both topic and format, with students producing research projects in both traditional and nontraditional forms. The UAs serve as research assistants for a study on cultural competence on campus; work on projects such as the Community Conversations workshops where they assist in facilitating conversations with students about diversity, inclusion, and social justice; assist with developing lesson plans for a cultural competence course taught for credit on campus; and coordinate programs in faculty and staff mentorship as well as peer mentorship. Through the UAP, they can collaboratively develop programming and outreach to serve diverse students, through which they increase their own cultural competence. The program also assesses students' attainment of cultural competency through a performance evaluation process that examines the effects of the research experience and engagement in content concerning differentness, analyzing their role in student success.

\section{Engaging Academically At-Risk Student Populations in Research: Initial Assessment of an Undergraduate Research Certificate Program}

Frances Shen, Keenan Dungey, Brian Kahn, John Laubersheimer, Tarah Sweeting-Trotter, Alexander Skarr, Sudeep

Sharma, Ben Walsh, and Holly Kent

University of Illinois Springfield, fshen3@uis.edu

\section{doi: $10.18833 /$ spur/1/3/3}

Since the University of Illinois Springfield (UIS) has been successful at recruiting a larger and more diverse undergraduate population, including academically at-risk students, more effort is needed to ensure their academic success. Thus, in collaboration with the Center for Academic Success in the Division of Academic Affairs, the Undergraduate Research Support program developed an Undergraduate Research Certificate program, seeking to encourage academically underprepared students to engage in undergraduate research by providing training and support services. The program is in the pilot stage with first-year students in the Students Transitioning for Academic Retention and Success (STARS) living-learning community. STARS serves an academically underprepared student population that typically consists of minorities and first-generation college students.

In the freshmen seminar course, STARS students participate in a number of workshops that address topics such as the definition of research, benefits of engaging in undergraduate research, undergraduate research resources and opportunities at UIS, presentation skills, information literacy skills, writing and citation skills, and so forth. Students also complete additional workshops outside of the course to receive the undergraduate research certificate. This requirement helps ensure that only students who are intrinsically motivated to engage in undergraduate research receive the certificate and matriculate into research opportunities that involve direct work with faculty members.

The program expressly involves undergraduate students who have actively engaged in research across various disciplines, as they participate as workshop presenters. This particular approach serves to (1) heighten students' interest in and motivation for undergraduate research through peer explanations of benefits and enthusiasm about research, (2) increase students' self-efficacy by witnessing peer success in undergraduate research, (3) enhance the visibility of student researchers on campus, and (4) involve students in a culture of undergraduate research engagement.

Pretest and posttests were administered to examine student perceptions of research self-efficacy, perceived barriers, and interest in undergraduate research. Results from the pretest confirm that undergraduate students often lack selfconfidence, awareness of opportunities, and the necessary skills to foster involvement in undergraduate research. Limited posttest data suggests the certificate program had a minor, positive effect on student perceptions. However, more data are needed to assess the effectiveness of the program. In the future, tracking student participation in undergraduate research after completion of the certificate is planned, as well as comparing outcomes for these students with those of similar academic backgrounds who do not participate in the program.

\section{Increasing Potential and Widening Horizons: Promoting Undergraduate Research at a Two-Year Regional Campus}

\author{
Elizabeth Easley and Sarah Hunt Sellhorst \\ University of South Carolina Lancaster, Sehunt@mailbox.sc.edu
}

\section{doi: $10.18833 /$ spur/1/3/4}

The University of South Carolina Lancaster (USCL)'s Research Club is a student organization established in 2015 as part of efforts to nurture a culture of scholarly 